\title{
Identificação Automática de Estilos de Aprendizagem: Uma Revisão Sistemática da Literatura
}

\section{Edilaine Santiago de Oliveira ${ }^{1}$, Gilvandenys Leites Sales ${ }^{1}$, Pryscilla de Sousa Pereira ${ }^{1}$, Ramires do Nascimento Moreira ${ }^{2}$}

\author{
${ }^{1}$ Instituto Federal de Educação, Ciência e Tecnologia do Ceará - \\ Programa de Pós-Graduação em Ciência da Computação (PPGCC) \\ Av. Treze de Maio, 2081 - Benfica - 60.040-215 - Fortaleza - CE - Brasil \\ ${ }^{2}$ Instituto Federal de Educação, Ciência e Tecnologia do Ceará - \\ Departamento de Engenharia de Telecomunicações \\ Av. Treze de Maio, 2081 - Benfica - 60.040-215 - Fortaleza - CE - Brasil \\ \{edilaine.santiago9,pry.spereira, denyssales, ramires.nas\}@gmail.com
}

\begin{abstract}
The spread and evolution of technology have revolutionized the way education is approached. The classification of learning styles within Virtual Learning Environments (VLE's) can help in the use of new teaching strategies based on the student profile, besides contributing to the personalization of these virtual environments. This research consists of a Systematic Review of Literature (SRL) of relevant works in the last four years that adopt automatic approaches, such as data mining and machine learning, in order to classify student's learning styles. In all, 12 papers were selected, considered significant for this research, for a more careful analysis. The results show that different techniques are applied, as well as different models of learning styles available in the literature.
\end{abstract}

Resumo. A disseminação e a evolução da tecnologia têm revolucionado a maneira de como a educação é abordada. A classificação dos estilos de aprendizagem dentro de Ambientes Virtuais de Aprendizagem (AVA's) pode auxiliar no uso de novas estratégias de ensino baseadas no perfil do aluno, além de contribuir na personalização desses ambientes virtuais. Esta pesquisa consiste em uma Revisão Sistemática da Literatura (RSL) de trabalhos relevantes nos últimos quatro anos que adotam abordagens automáticas, como mineração de dados e aprendizado de máquina, para classificação dos estilos de aprendizagem dos alunos. Ao todo foram selecionados 12 artigos, considerados significativos para esta pesquisa, para realização de uma análise mais criteriosa. Os resultados mostram que diferentes técnicas são aplicadas, assim como diferentes modelos de estilos de aprendizagem disponíveis na literatura.

\section{Introdução}

A disseminação e a evolução da tecnologia, que está cada vez mais presente em nosso meio, revolucionam a maneira como a educação é abordada. Essa revolução mudou a forma de aprender, tornando os indivíduos mais ativos na aprendizagem, buscando mais interatividade e participação [Macedo 2010]. A modalidade de ensino de Educação a 
Distância $(\mathrm{EaD})$ é uma prova de como a tecnologia afeta na educação, visto que a partir desta é possível atingir um maior número de pessoas interconectadas por meio da internet. Gradativamente as instituições de ensino têm adotado o uso de cursos a distância, é o que mostra a pesquisa elaborada pelo Instituto Nacional de Estudos e Pesquisas Educacionais Anísio Teixeira [INEP 2016].

Estes cursos utilizam como principal recurso os Ambientes Virtuais de Aprendizagem (AVA's), também conhecidos como Learning Management System (LMS). Estes ambientes podem ser compreendidos como espaços pedagógicos colaborativos dispostos na Internet para a formação de comunidades de aprendizes [Sales 2010]. Seja dentro ou fora desses ambientes uma difícil tarefa encontrada pelo professor é conseguir adaptar os métodos de aprendizagem para diferentes tipos de alunos [Felder and Brent 2005]. A falta de encontros presenciais provavelmente irá dificultar ainda mais a percepção de alguns aspectos comportamentais dos alunos. Outro obstáculo relacionado a estes ambientes virtuais é que eles tendem a ser centrados no curso, ao invés de serem focados no aluno [Graf and List 2005].

Esta abordagem pode não favorecer no desempenho dos alunos, onde cada um tem seus pontos fortes e fracos, interesses, níveis de motivação e abordagens diferentes para estudar [Henze et al. 2004]. A diversidade de tipos de personalidades, assim como os impactos dos estados cognitivos na compreensão e resolução de desafios, mostram a importância do processo de aprendizagem estar alinhado com os traços da personalidade de cada aluno [Farias et al. 2014].

Assim, a identificação dos estilos de aprendizagem dentro de um AVA pode auxiliar na construção de sistemas personalizados que mesclam os benefícios dos sistemas de aprendizagem com uma abordagem adaptada para cada estilo. Essas plataformas de $e$ learning personalizadas buscam melhorar a satisfação e a motivação dos alunos ao longo do curso [Halawa et al. 2015a].

Portanto, considerando a importância do tema proposto, decidiu-se realizar uma Revisão Sistemática da Literatura (RSL) a fim de identificar as estratégias utilizadas para classificação automática de perfis de aprendizagem, assim como detectar os modelos de estilos mais comuns. O objetivo é avaliar e interpretar os estudos relevantes nessa área e, consequentemente, levantar conteúdo que auxilie equipes responsáveis por novas modelagens de sistemas adaptáveis com base no perfil de aprendizagem do aluno.

O restante deste trabalho está organizado da seguinte maneira: na seção 2 abordamos a metodologia desta pesquisa, explicando todas as etapas seguidas no protocolo de revisão. Na seção 3 apresentamos os resultados obtidos após os critérios de inclusão e exclusão, assim como a síntese e análise dos artigos selecionados. Por fim, na seção 4 apresentamos as conclusões levantadas por intermédio desta pesquisa.

\section{Metodologia}

Para atingir o objetivo desta pesquisa, foram realizadas as seguintes etapas (Figura 1), conforme indicado por [Kitchenham 2004].

\subsection{Planejamento}

Inicialmente foram definidas duas questões de pesquisa que esta RSL tem como objetivo responder: 
Figura 1. Etapas da metodologia

- QP1: Quais modelos de estilos de aprendizagem da literatura são mais utilizados nos estudos realizados?

- QP2: Quais são os diferentes métodos usados para identificar estilos de aprendizagem de forma automática?

O protocolo de revisão foi elaborado de forma a responder às questões de pesquisas definidas. No mês de março de 2018 foram consultadas quatro bases de arquivos científicos para a etapa de seleção dos trabalhos: Scopus, Association for Computing Machinery Digital Library (ACM DL), Web of Science e IEEEXplorer.

Estas bases foram selecionadas devido aos mecanismos de buscas online que possuem, possibilitando o uso de filtros por ano e tipo de publicação, além de abranger pesquisas relacionadas ao tema da computação. As strings de busca utilizadas nestas bases (Tabela 1) foram elaboradas a partir de palavras-chaves como: data mining methods, data mining techniques, machine learning, learning styles, assim como seus sinônimos.

Tabela 1. Strings de busca usadas nos repositórios

\begin{tabular}{|l|l|}
\hline \multicolumn{1}{|c|}{ Repositório } & \multicolumn{1}{c|}{ String de Busca } \\
\hline Scopus & $\begin{array}{l}\text { TITLE-ABS-KEY ( ( "learning styles" OR } \\
\text { "learning style" ) AND ( "Data mining } \\
\text { techniques" OR "Data mining methods" OR } \\
\text { "data mining" OR "machine learning")) }\end{array}$ \\
\hline ACM & $\begin{array}{l}\text { Searched for +("learning style") +(" Data mining } \\
\text { techniques" "Data mining methods" "data } \\
\text { mining" "machine learning") }\end{array}$ \\
\hline Web of Science & $\begin{array}{l}\text { TS=(("learning style*") AND ( "Data mining } \\
\text { techniqu*" OR "Data mining method*” OR } \\
\text { "data mining" OR "machine learning")) }\end{array}$ \\
\hline IEEEXplorer & $\begin{array}{l}\text { (((("learning styles") AND "Data mining } \\
\text { techniques") OR "Data mining methods") OR } \\
\text { "data mining") OR "machine learning") }\end{array}$ \\
\hline
\end{tabular}

Para que a pesquisa seja mais específica e criteriosa, somente foram aceitos trabalhos que atendessem aos critérios de inclusão e exclusão definidos a seguir (Tabela 2). Nesta revisão desejamos explorar artigos mais recentes para identificar quais técnicas de 
classificação e quais modelos de estilos de aprendizagem são mais utilizados atualmente, logo é importante ressaltar que apenas foram consideradas publicações realizadas nos últimos quatro anos. Ao todo foram selecionados cinco critérios de inclusão e quatro de exclusão, utilizados na etapa de seleção.

Tabela 2. Critérios de Inclusão e Exclusão

\begin{tabular}{|l|l|l|}
\hline \multicolumn{1}{|c|}{ Critério } & ID & \multicolumn{1}{c|}{ Descrição } \\
\hline \multirow{5}{*}{ Inclusão } & I-1 & Trabalhos na área de Ciências da Computação \\
\cline { 2 - 4 } & I-2 & Trabalhos escritos em inglês \\
\cline { 2 - 4 } & I-3 & Trabalhos publicados entre 2014 e março de 2018 \\
\cline { 2 - 4 } & I-4 & Artigos revisados por pares \\
\cline { 2 - 4 } & I-5 & $\begin{array}{l}\text { Utiliza abordagens automáticas para identificar os } \\
\text { estilos de aprendizagem }\end{array}$ \\
\hline \multirow{5}{*}{ Exclusão } & E-1 & Trabalhos duplicados \\
\cline { 2 - 4 } & E-2 & $\begin{array}{l}\text { Trabalhos que não contém palavras-chaves no título } \\
\text { ou resumo }\end{array}$ \\
\cline { 2 - 4 } & E-3 & Número de páginas menor que quatro \\
\cline { 2 - 4 } & E-4 & $\begin{array}{l}\text { Trabalhos não disponíveis integralmente na web } \\
\text { e não acessiveis de forma gratuita }\end{array}$ \\
\hline
\end{tabular}

\subsection{Execução}

A etapa de execução realiza a identificação, seleção e extração dos trabalhos. Durante o processo de identificação, as strings de busca foram aplicadas nas bases de artigos científicos e o conjunto inicial foi de 566 artigos, distribuídos nos quatro repositórios (Figura 2).

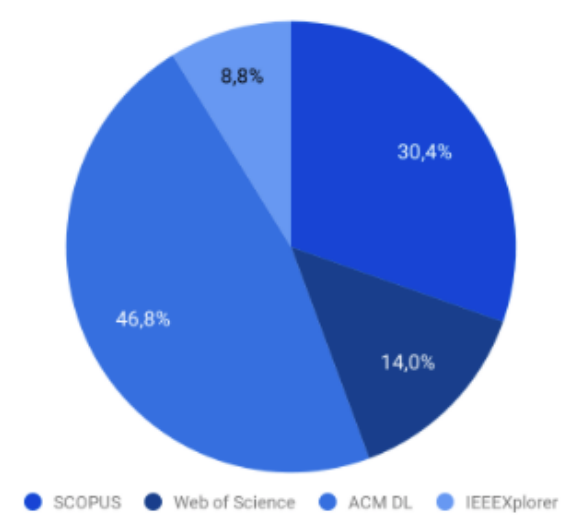

Figura 2. Artigos selecionados por repositório

A maior parte dos artigos (77,2\%) foi encontrada nas bases de dados da ACM DL e Scopus, onde quase metade $(46,8 \%)$ dos artigos vieram da ACM DL. Os outros $22,8 \%$ dos trabalhos foram encontrados nos repositórios do IEEEXplorer e Web of Science, onde somente $8,8 \%$ vieram do IEEEXplorer.

$\mathrm{Na}$ etapa de seleção ocorre a aplicação dos critérios de inclusão e exclusão. A fim de encontrar quais artigos utilizam abordagens automáticas para identificar os estilos 
de aprendizagem, realizamos a leitura do título e resumo dos noventa artigos finais, onde apenas doze trabalhos foram escolhidos para uma análise completa (Figura 3). Estes trabalhos são analisados de forma mais detalhada na próxima seção a fim de responder às questões de pesquisa elaboradas.

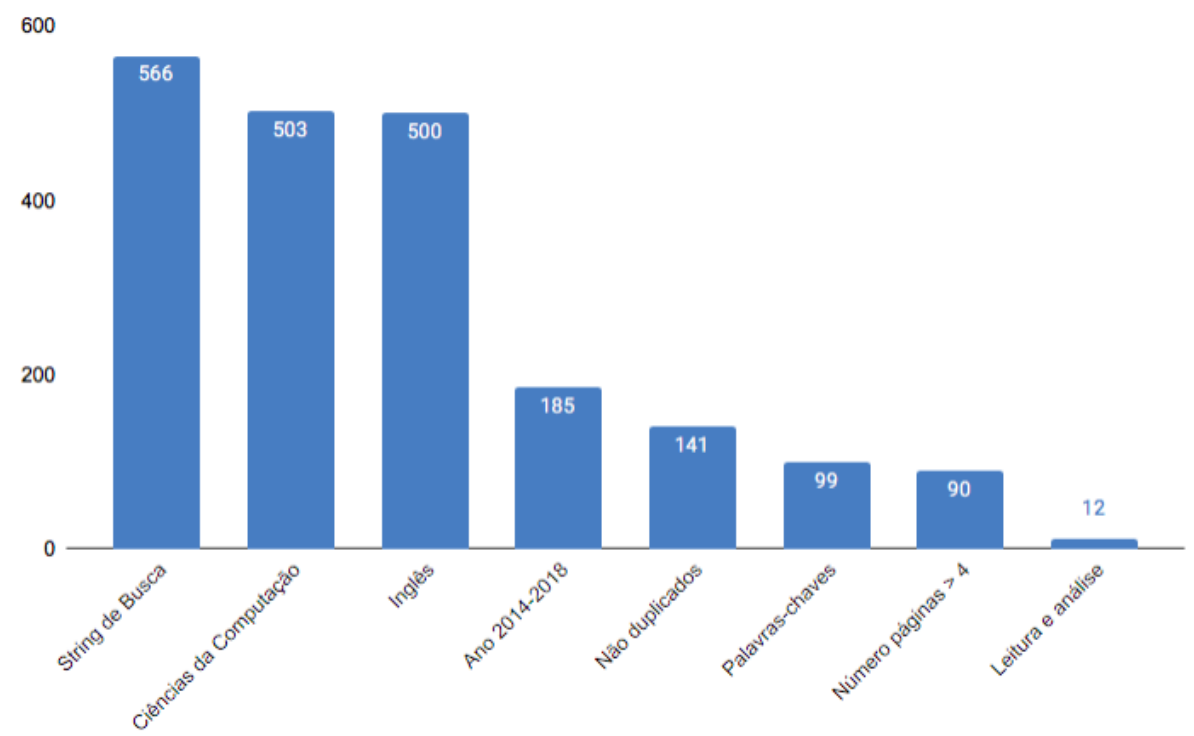

Figura 3. Aplicação dos critérios de inclusão e exclusão

Com a aplicação do primeiro critério de inclusão (somente artigos da área da computação), 63 artigos foram excluídos. Dos 503 artigos que ficaram, somente 3 não são da língua inglesa. Na aplicação do critério de inclusão 3 (trabalhos publicados entre 2014 e março de 2018), 185 artigos foram extraídos para aplicação dos outros critérios.

Após retirar os trabalhos duplicados, trabalhos que não contém palavras-chaves no resumo ou título e trabalhos com número de páginas menor que quatro, foram selecionados 12 artigos para a etapa de análise e avaliação. Nesta etapa levamos em consideração também a qualidade de cada trabalho, assim como a qualidade da conferência/periódico onde os mesmos foram publicados.

Estes trabalhos foram selecionados para serem lidos por completo, de modo a avaliar se realmente descrevem a forma, com alto nível de detalhe, de como é feita a classificação dos estilos de aprendizagem nos ambientes virtuais de aprendizagem.

\section{Discussão e Análise dos Resultados}

Esta seção apresenta os resultados produzidos pela extração de dados dos estudos primários de acordo com o processo descrito na Seção 2. Para fornecer uma maneira mais simples de visualizar os resultados, estes foram separados em dois grupos: informações gerais e respostas das questões de pesquisa. Em primeiro lugar, apresentamos uma visão geral dos estudos relevantes, em seguida as respostas às questões de pesquisa são apresentadas.

\subsection{Informações Gerais dos Resultados}

Os documentos filtrados foram lidos e, em seguida, recuperamos muitas outras informações. Depois disso, armazenamos o título, ano de publicação, local de publicação 
(periódico ou conferência), nome e localização do autor. Inicialmente consideramos algumas informações gerais, como, distribuição de artigos por ano (Tabela 3).

Tabela 3. Distribuição de artigos por ano

\begin{tabular}{|c|l|l|}
\hline Ano & \multicolumn{1}{|c|}{ Artigo } & Publicação \\
\hline \multirow{2}{*}{2014} & {$[$ Efrati et al. 2014] } & Conferência \\
\cline { 2 - 3 } & {$[$ Mohamed et al. 2014] } & Conferência \\
\hline \multirow{4}{*}{2015} & {$[$ Halawa et al. 2015a] } & Conferência \\
\cline { 2 - 3 } & {$[$ Hassan and Hegazy 2015] } & Conferência \\
\cline { 2 - 3 } & {$[$ Paireekreng and Prexawanprasut 2015] } & Conferência \\
\cline { 2 - 3 } & {$[$ Sweta and Lal 2015] } & Conferência \\
\hline \multirow{4}{*}{2016} & {$[$ Adel et al. 2016] } & Conferência \\
\cline { 2 - 3 } & {$[$ Hung et al. 2016] } & Periódico \\
\cline { 2 - 3 } & {$[$ Liyanage et al. 2016] } & Periódico \\
\cline { 2 - 3 } & {$[$ Popescu et al. 2016] } & Conferência \\
\hline \multirow{2}{*}{2017} & {$[$ Binh and Duy 2017] } & Conferência \\
\cline { 2 - 3 } & {$[$ Zhong et al. 2017] } & Periódico \\
\hline
\end{tabular}

Os seguintes padrões foram observados no resultado: o número de publicações varia de 2 a 4 artigos; 2015 e 2016 são os anos mais produtivos com 4 publicações cada. A maioria dos estudos (75\%) foi publicada em anais de congressos, enquanto os trabalhos [Hung et al. 2016, Liyanage et al. 2016, Zhong et al. 2017], foram publicados em periódicos.

Já as informações sobre em quais países foram realizadas as pesquisas filtradas, são exibidas na Tabela 4. Uma tendência que se observa nos resultados é que, com exceção do Egito, todos os trabalhos são de países diferentes, o que pode apontar para existência de particularidades locais para o tema de perfis de aprendizagem.

Tabela 4. Países das pesquisas selecionadas

\begin{tabular}{|l|l|}
\hline \multicolumn{1}{|c|}{ Localização Autor } & \multicolumn{1}{c|}{ Artigo } \\
\hline China & [Zhong et al. 2017] \\
\hline Itália & [Efrati et al. 2014] \\
\hline Egito & [Hassan and Hegazy 2015], [Halawa et al. 2015a] \\
\hline Tailândia & [Paireekreng and Prexawanprasut 2015] \\
\hline Malásia & [Mohamed et al. 2014] \\
\hline Japão & [Liyanage et al. 2016] \\
\hline Taiwan & [Hung et al. 2016] \\
\hline Romênia e Estados Unidos & [Popescu et al. 2016] \\
\hline Vietnã & [Binh and Duy 2017] \\
\hline Reino Unido & [Adel et al. 2016] \\
\hline Índia & [Sweta and Lal 2015] \\
\hline
\end{tabular}




\subsection{Respostas para as Questões de Pesquisa}

Nesta seção nós apresentamos os resultados agrupados para responder as duas questões de pesquisas levantadas no início deste documento. O processo foi iniciado com a leitura dos doze artigos selecionados. Inicialmente foi feita a identificação dos objetivos e das metodologias de cada um dos artigos, selecionando informações relevantes para responder cada uma das questões.

QP1: Quais modelos de estilos de aprendizagem da literatura são mais utilizados nos estudos realizados?

Diversos tipos de modelos de estilos de aprendizagem foram abordados nos trabalhos selecionados (Tabela 5). O modelo de Felder e Silverman foi o mais utilizado nas pesquisas, totalizando $66 \%$ dos trabalhos. Comparando-se o número de citações deste modelo com os demais, através do Google Scholar, também nota-se uma maior tendência em seu uso.

Tabela 5. Modelos de Estilos de Aprendizagem

\begin{tabular}{|l|l|}
\hline \multicolumn{1}{|c|}{ Modelo } & \multicolumn{1}{c|}{ Artigo } \\
\hline Felder-Silverman & $\begin{array}{l}\text { [Adel et al. 2016], [Binh and Duy 2017], [Hung et al. 2016], } \\
\text { [Liyanage et al. 2016], [Mohamed et al. 2014], } \\
\text { [Paireekreng and Prexawanprasut 2015], } \\
\text { [Popescu et al. 2016],[Sweta and Lal 2015] }\end{array}$ \\
\hline Grasha-Riechmann & [Efrati et al. 2014] \\
\hline Honey-Mumford & [Zhong et al. 2017] \\
\hline Myers-Briggs & [Halawa et al. 2015a] \\
\hline VARK & [Hassan and Hegazy 2015] \\
\hline
\end{tabular}

No modelo de Felder e Silverman (FSLSM) os estilos de aprendizagem são definidos como as preferências dos alunos na forma de perceber, captar, organizar, processar e compreender a informação [Felder and Silverman 1988]. Neste modelo os alunos são categorizados em quatro dimensões e cada dimensão está dividida em dois estilos (ativo/reflexivo, sensorial/intuitivo, visual/verbal e sequencial/global).

De acordo com o modelo FSLSM, alunos ativos preferem trabalhos em equipe, experimentações e discussões. Já o tipo reflexivo prefere trabalhos individuais, conceito e teorias. Na dimensão de percepção, o estilo sensorial prefere obter informações por meio de dados experimentais e sentidos (vendo, ouvindo, tocando), enquanto que o perfil intuitivo prefere abstrações e definições.

Com relação à forma que os indivíduos captam as informações no modelo FSLSM, perfis visuais preferem captar a informação por meio de imagens, gráficos, vídeos, enquanto perfis verbais preferem textos e áudios. Por último temos a dimensão de organização, que indica como o indivíduo prefere progredir, podendo ser em sequência, onde tendem a progredir de forma lógica ou globalmente, onde preferem ter a visão geral do todo, aprendendo em saltos e com mais liberdade. O uso frequente desse modelo se da ao fato dele ter sido baseado em extensa experimentação, que validou as dimensões em uma população de estudantes de engenharia [Dorça 2012]. 
Dos oito trabalhos que abordam o modelo de Felder e Silverman, seis desses trabalhos [Paireekreng and Prexawanprasut 2015, Liyanage et al. 2016, Hung et al. 2016, Popescu et al. 2016, Binh and Duy 2017, Adel et al. 2016], usam o questionário Index Learning System (ILS), elaborado por Felder e Soloman [Felder and Soloman 1997] para identificar os estilos de aprendizagem dos alunos. Este questionário contém 44 questões objetivas, onde para cada dimensão existem 11 questões equivalentes, cada uma com duas opções de escolha. Os autores fazem uso das respostas do questionário para comparar com a classificação feita pelas abordagens automáticas que utilizam os registros das interações dos alunos com o ambiente virtual.

A pesquisa de [Efrati et al. 2014] sugere o uso do modelo Grasha-Riechmann, aplicado como referência para classificar os estilos. Este modelo possui apenas três dimensões: intra-subjetividade/intersubjetividade, competitivo/colaborativo, independente/dependente, onde cada uma possui uma escala de valores, podendo ser do tipo baixo, médio ou alto. Grasha e Riechmann revisaram e avaliaram os estilos de aprendizagem de estudantes universitários por meio de uma perspectiva social, a fim de identificar diferentes abordagens no ambiente da sala de aula [Grasha 1972].

Outro modelo utilizado foi o de Honey e Mumford, no trabalho de [Zhong et al. 2017]. Este modelo possui quatro estilos: reflexivo, teórico, pragmático e ativo. Ele é derivado diretamente da teoria de [Kolb 1984]. Já o trabalho de [Halawa et al. 2015b] aborda o modelo de Myers-Briggs, que utiliza o Myers-Briggs Type Indicator (MBTI), onde este avalia tipos de personalidade e preferências por meio de quatro aspectos da personalidade: extroversão/introversão, detecção/intuição, pensamento/sentimento, julgando/percebendo. A combinação dessas diferentes preferências resulta na possibilidade de 16 diferentes tipos de estilos de aprendizagem, assim como no modelo de Felder e Silverman.

Por último temos o modelo de VARK, acrônimo para Visual, Auditory, Read/write, e Kinesthetic, utilizado no trabalho de [Hassan and Hegazy 2015]. Esse modelo também utiliza um questionário, onde este é usado para categorizar quatro modalidades de preferências sensoriais: visual, auditivo, ler/escrever e cinestésica. Nesta última modalidade o aluno adquire conhecimento através de experiência simulada ou real. Logo, nesta classificação, o aluno pode ter preferência em um único tipo de perfil ou ser do tipo multimodal, onde terá preferência em mais de um estilo, totalizando até 15 possibilidades de estilos diferentes.

\section{QP2: Quais são os diferentes métodos usados para identificar estilos de aprendizagem de forma automática?}

Inicialmente identificamos e apresentamos a distribuição dos tipos de técnicas usadas nos trabalhos referentes à identificação dos estilos de aprendizagem (Figura 4). A maioria das pesquisas $(71,4 \%)$ implementa a técnica de classificação. Esse método é um tipo de aprendizado de máquina supervisionado [Duda et al. 2000]. 


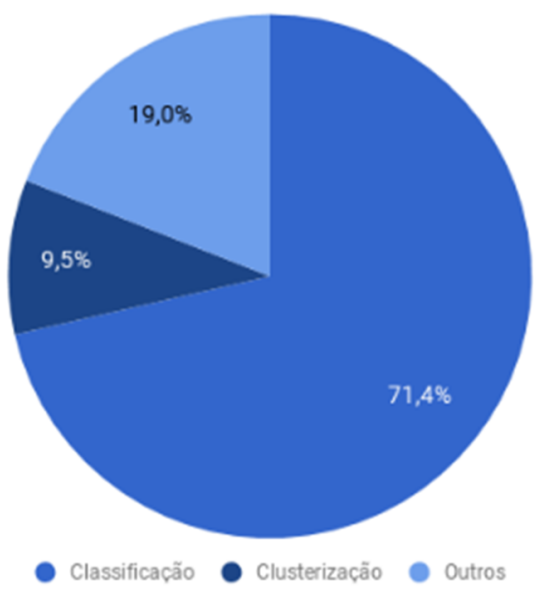

Figura 4. Técnicas usadas para identificação dos estilos de aprendizagem

Ao todo, vinte algoritmos foram utilizados nesses trabalhos (Tabela 6). De todos esses, os algoritmos de Redes Bayesianas, Árvores de Decisão, Naive Bayes e Redes Neurais Artificiais (RNA), foram os mais referenciados nas pesquisas, onde as Redes Bayesianas foram aplicadas em cinco trabalhos e os demais em quatro. Apesar dessas técnicas não serem tão recentes, ainda são aplicáveis na classificação do perfil dos estudantes na atualidade.

Já os outros algoritmos foram usados em um ou dois trabalhos apenas. Dos doze artigos analisados, cinco deles [Efrati et al. 2014, Popescu et al. 2016, Hung et al. 2016, Binh and Duy 2017, Sweta and Lal 2015], utilizaram somente um algoritmo, enquanto os demais utilizaram no mínimo dois [Zhong et al. 2017] e no máximo dez [Halawa et al. 2015b].

Tabela 6. Algoritmos utilizados

\begin{tabular}{|l|l|l|}
\hline \multicolumn{1}{|c|}{ Algoritmo } & \multicolumn{1}{c|}{ Artigo } & \multicolumn{1}{c|}{ Acurácia } \\
\hline Bayesian Network & $\begin{array}{l}\text { [Hassan and Hegazy 2015], [Liyanage et al. 2016], [Halawa et al. 2015a], } \\
\text { [Adel et al. 2016], [Sweta and Lal 2015] }\end{array}$ & $89,9 \%, 68,1 \%, 88,5 \%, 83,3 \%,-$ \\
\hline Bijective Soft Set & [Mohamed et al. 2014] & $98,4 \%$ \\
\hline Decision Table & [Halawa et al. 2015a] & $85,4 \%$ \\
\hline Decision Tree/J48 Graft & $\begin{array}{l}\text { [Paireekreng and Prexawanprasut 2015], [Liyanage et al. 2016], } \\
\text { [Halawa et al. 2015a], [Adel et al. 2016] }\end{array}$ & $62,7 \%, 79,1 \%, 92,1 \%, 83,3 \%$ \\
\hline Discriminant Function Analysis (DFA) & [Popescu et al. 2016] & $62,6 \%$ \\
\hline Expectation Maximization (EM) & [Efrati et al. 2014] & - \\
\hline FURIA & [Mohamed et al. 2014] & $97,2 \%$ \\
\hline Hyper Pipes & [Hassan and Hegazy 2015] & $92,3 \%$ \\
\hline JRIP & [Halawa et al. 2015a], [Hassan and Hegazy 2015] & $63,5 \%, 90,3 \%$ \\
\hline K-means & [Zhong et al. 2017], [Hung et al. 2016] & $56,5 \%,-$ \\
\hline KNN/IBK & [Halawa et al. 2015a], [Adel et al. 2016], [Hassan and Hegazy 2015] & $90,6 \%, 62,9 \%, 97 \%$ \\
\hline KStar & [Halawa et al. 2015a] & $48,9 \%$ \\
\hline Naive Bayes & $\begin{array}{l}\text { [Halawa et al. 2015a], [Paireekreng and Prexawanprasut 2015], } \\
\text { [Liyanage et al. 2016], [Adel et al. 2016] }\end{array}$ & $68,2 \%, 42,5 \%, 65,2 \%, 79,6 \%$ \\
\hline OneR & [Halawa et al. 2015a] & $97,4 \%$ \\
\hline RandomTree/Random Forests & [Liyanage et al. 2016], [Halawa et al. 2015a], [Adel et al. 2016] & $78,1 \%, 93,2 \%, 81,6 \%$ \\
\hline Rede Neural/MLP & $\begin{array}{l}\text { [hong et al. 2017], [Paireekreng and Prexawanprasut 2015], } \\
\text { [Binh and Duy 2017], [Adel et al. 2016] }\end{array}$ & $100 \%, 88,3 \%, 80,6 \%, 69,9 \%$ \\
\hline Rough Set & [Mohamed et al. 2014] & $96,3 \%$ \\
\hline Simple CART & [Adel et al. 2016] & $83,3 \%$ \\
\hline Simple Logistic & [Hassan and Hegazy 2015] & $93,8 \%$ \\
\hline SVM & [Zhong et al. 2017], [Paireekreng and Prexawanprasut 2015] & $99,7 \%, 25,5 \%$ \\
\hline
\end{tabular}


Os algoritmos que obtiveram as melhores acurácias foram: RNA (100\%), SVM $(99,7 \%)$ [Zhong et al. 2017], Bijective Soft Set $(98,4 \%)$ [Mohamed et al. 2014], OneR $(97,4 \%)$ [Halawa et al. 2015a] e IBK (97\%) [Hassan and Hegazy 2015].

Metade dos trabalhos analisados [Efrati et al. 2014, Hassan and Hegazy 2015, Liyanage et al. 2016, Halawa et al. 2015a, Adel et al. 2016, Sweta and Lal 2015], utilizou a ferramenta Waikato Environment for Knowledge Analysis (WEKA) para aplicação e análise dos algoritmos. Essa ferramenta possui uma coleção de algoritmos de aprendizado de máquina para mineração de dados, além de ser uma plataforma de código aberto baseada em Java desenvolvida na Universidade de Waikato, Nova Zelândia [Liyanage et al. 2016]. Desses trabalhos que fizeram uso da ferramenta WEKA, cinco deles usaram o ambiente virtual Modular Object Oriented Developmental Learning Environment (Moodle), totalizando $42 \%$ dos doze trabalhos pesquisados.

Já os dados mais utilizados para fazer a identificação dos estilos de aprendizagem, foram dados coletados a partir dos registros gerados pelas interações dos alunos dentro dos AVAs [Efrati et al. 2014, Zhong et al. 2017, Hassan and Hegazy 2015, Liyanage et al. 2016, Hung et al. 2016, Halawa et al. 2015a, Sweta and Lal 2015]. Dois trabalhos [Popescu et al. 2016, Adel et al. 2016] usaram análise de dados textuais, um utilizou somente dados do questionário ILS [Binh and Duy 2017], outro usou dados pessoais [Paireekreng and Prexawanprasut 2015] e o último usou dados simulados das interações no AVA [Mohamed et al. 2014].

Abaixo apresentamos o resumo de todas as características extraídas dos trabalhos (tabela 7) .

Tabela 7. Síntese dos trabalhos selecionados

\begin{tabular}{|c|c|c|c|c|c|c|}
\hline Autor & Modelo & Algoritmo & Dados Utilizados & Qtde. Alunos & Ambiente Virtual & Ferramenta \\
\hline [Adel et al. 2016] & Felder-Silverman & $\begin{array}{l}\text { Naive Bayes, Bayes } \\
\text { Network, J48, } \\
\text { Simple CART, Random Forest, } \\
\text { IBK e RBF }\end{array}$ & $\begin{array}{l}\text { ILS e Registros do sistema } \\
\text { de tutoria inteligente }\end{array}$ & 75 & Oscar & WEKA \\
\hline [Binh and Duy 2017] & Felder-Silverman & MLP & ILS & 316 & - & Phyton \\
\hline [Efrati et al. 2014] & Grasha-Riechmann & Expectation Maximization (EM) & $\begin{array}{l}\text { Registros do ambiente } \\
\text { virtual }\end{array}$ & - & Moodle & WEKA \\
\hline [Halawa et al. 2015a] & Myers-Briggs & $\begin{array}{l}\text { NaiveBayes, BayesNet, Kstar, } \\
\text { Random forest, } \\
\text { J48, OneR, JRIP, KNN/IBK, } \\
\text { RandomTree e Decision Table. }\end{array}$ & $\begin{array}{l}\text { Registros do ambiente } \\
\text { virtual e Redes Sociais }\end{array}$ & 240 & Moodle & WEKA \\
\hline [Hassan and Hegazy 2015] & VARK & $\begin{array}{l}\text { Simple Logistic, IBK, Bayes Net, } \\
\text { J48 Graft, Hyper Pipes, JRIP }\end{array}$ & $\begin{array}{l}\text { Registros do ambiente } \\
\text { virtual e Redes Sociais }\end{array}$ & 131 & Moodle & WEKA \\
\hline [Hung et al. 2016] & Felder-Silverman & K-means & $\begin{array}{l}\text { ILS e Registros do } \\
\text { ambiente virtual (jogo) }\end{array}$ & 67 & - & - \\
\hline [Liyanage et al. 2016] & Felder-Silverman & $\begin{array}{l}\text { J48, Bayesian Network, } \\
\text { Naive Bayes e Random Forests. }\end{array}$ & $\begin{array}{l}\text { ILS e Registros do } \\
\text { ambiente virtual }\end{array}$ & 80 & Moodle & WEKA \\
\hline [Mohamed et al. 2014] & Felder-Silverman & $\begin{array}{l}\text { Bijective Soft Set, FURIA } \\
\text { e Rough Set }\end{array}$ & $\begin{array}{l}\text { Dados simulados } \\
\text { de registros do ambiente virtual }\end{array}$ & - & - & - \\
\hline [Paireekreng and Prexawanprasut 2015] & Felder-Silverman & $\begin{array}{l}\text { Decision Tree, Naive Bayes, } \\
\text { RNA e SVM }\end{array}$ & $\begin{array}{l}\text { ILS e Dados pessoais } \\
\text { dos alunos }\end{array}$ & 400 & - & - \\
\hline [Popescu et al. 2016] & Felder-Silverman & Discriminant Function Analysis (DFA) & ILS e Análise de texto & 66 & eMUSE & Phyton \\
\hline [Sweta and Lal 2015] & Felder-Silverman & Bayesian Network & Registros do ambiente virtual & 40 & Moodle & WEKA \\
\hline [Zhong et al. 2017] & Honey e Mumford & K-means, SVM, Rede Neural Artificial & $\begin{array}{l}\text { ILS e Registros do } \\
\text { ambiente virtual }\end{array}$ & 183 & MOOC & - \\
\hline
\end{tabular}

\section{Conclusão}

Neste artigo apresentamos uma revisão sistemática da literatura para analisar os trabalhos que usam diferentes abordagens automáticas na identificação dos estilos de aprendizagem de alunos. Procuramos trabalhos de 2014 a 2018 e definimos questões de pesquisa juntamente com um protocolo de revisão para orientar nossa busca feita em quatro bases de artigos. Pretende-se com esse estudo ampliar o estado da arte, contribuindo por evidenciar trabalhos de pesquisa voltados para identificação desses estilos. 
As principais conclusões são de que a maioria dos trabalhos são de conferências, o modelo Felder e Silverman é o modelo mais utilizado, as técnicas mais utilizadas são do tipo classificação, onde as mais referenciadas foram: RNA, Árvores de decisão, Redes Bayesianas e Naive Bayes. Outro ponto que merece destaque nos resultados encontrados é a predominância de dois tipos de coleta de dados: as coletas baseadas em questionários e as coletas baseadas em registros do ambiente virtual de aprendizagem.

Com isso, além das técnicas/ferramentas utilizadas presentes nos estudos selecionados, esta pesquisa mostrou os avanços no que se refere ao uso de técnicas de mineração de dados educacionais para identificação dos perfis de aprendizagem. Espera-se a partir desta revisão estimular a condução de mais pesquisas nessa área, de modo a potencializar o uso de novos algoritmos de classificação, além de estimular o desenvolvimento de ambientes virtuais personalizados com base nesses perfis.

A partir desta RSL, concluímos que identificação dos estilos de aprendizagem em AVA é uma linha de pesquisa que tem despertado o interesse da comunidade acadêmica e explorado uma diversidade de tecnologias.

\section{Agradecimentos}

Este pesquisa é apoiada pelo PPGCC/IFCE e pela Fundação Cearense de Apoio ao Desenvolvimento Científico e Tecnológico - FUNCAP.

\section{Referências}

Adel, N., Latham, A., and Crockett, K. A. (2016). Towards socially intelligent automated tutors: Predicting learning style dimensions from conversational dialogue. In 2016 Intl IEEE Conferences on Ubiquitous Intelligence Computing, Advanced and Trusted Computing, Scalable Computing and Communications, Cloud and Big Data Computing, Internet of People, and Smart World Congress (UIC/ATC/ScalCom/CBDCom/IoP/SmartWorld), pages 315-320.

Binh, H. T. and Duy, B. T. (2017). Predicting students' performance based on learning style by using artificial neural networks. In 2017 9th International Conference on Knowledge and Systems Engineering (KSE), pages 48-53.

Dorça, F. A. (2012). Uma abordagem estocástica baseada em aprendizagem por reforço para modelagem automática e dinâmica de estilos de aprendizagem de estudantes em sistemas adaptativos e inteligentes para educação a distância. Tese de doutorado, Universidade Federal de Uberlândia, Uberlândia, MG, Brasil.

Duda, R. O., Hart, P. E., and Stork, D. G. (2000). Pattern Classification (2Nd Edition). Wiley-Interscience.

Efrati, V., Limongelli, C., and Sciarrone, F. (2014). A data mining approach to the analysis of students' learning styles in an e-learning community: A case study. In Stephanidis, C. and Antona, M., editors, Universal Access in Human-Computer Interaction. Universal Access to Information and Knowledge, pages 289-300, Cham. Springer International Publishing.

Farias, A., Gomes, T., and Cabral, G. (2014). Uma proposta metodológica para o ensino de programação baseado na relação entre perfis cognitivos, padrões pedagógicos 
e autoregulação dos estudantes. XXXIV Congresso da Sociedade Brasileira de Computação, XXII Workshop sobre Educação em Computação.

Felder, R. M. and Brent, R. (2005). Understanding student differences. Journal of Engineering Education, 94(1):57-72.

Felder, R. M. and Silverman, L. K. (1988). Learning and teaching styles in engineering education. ENGINEERING EDUCATION.

Felder, R. M. and Soloman, B. A. (1997). Index of learning styles questionnaire.

Graf, S. and List, B. (2005). An evaluation of open source e-learning platforms stressing adaptation issues. In Fifth IEEE International Conference on Advanced Learning Technologies (ICALT'05), pages 163-165.

Grasha, A. F. (1972). Observations on relating teaching goals to student response styles and classroom methods. American Psychologist, 27(2):144-147.

Halawa, M. S., Shehab, M. E., and Hamed, E. M. R. (2015a). Predicting student personality based on a data-driven model from student behavior on $1 \mathrm{~ms}$ and social networks. In 2015 Fifth International Conference on Digital Information Processing and Communications (ICDIPC), pages 294-299.

Halawa, M. S., Shehab, M. E., and Hamed, E. M. R. (2015b). Predicting student personality based on a data-driven model from student behavior on $1 \mathrm{~ms}$ and social networks. In 2015 Fifth International Conference on Digital Information Processing and Communications (ICDIPC), pages 294-299.

Hassan, S. and Hegazy, A. E. F. (2015). A model recommends best machine learning algorithm to classify learners based on their interactivity with moodle. In $2015 \mathrm{Se}$ cond International Conference on Computing Technology and Information Management (ICCTIM), pages 49-54.

Henze, N., Dolog, P., and Nejdl, W. (2004). Reasoning and ontologies for personalized elearning in the semantic web. Journal of Educational Technology and Society, 7(4):8297.

Hung, Y. H., Chang, R. I., and Lin, C. F. (2016). Hybrid learning style identification and developing adaptive problem-solving learning activities. Computers in Human Behavior, 55:552 - 561 .

INEP (2016). Censo da Educação Superior 2016 - Notas Estatísticas. Instituto Nacional de Estudos e Pesquisas Educacionais Anísio Teixeira.

Kitchenham, B. (2004). Procedures for Undertaking Systematic Reviews.

Kolb, D. (1984). Experiential Learning: Experience As The Source Of Learning And Development, volume 1.

Liyanage, M. P. P., Gunawardena, K. L., and Hirakawa, M. (2016). Detecting learning styles in learning management systems using data mining. Journal of Information Processing, 24(4):740-749.

Macedo, C. M. S. (2010). Diretrizes para criação de objetos de aprendizagem acessíveis. Tese de doutorado, Universidade Federal de Santa Catarina, Florianópolis, SC, Brasil. 
Mohamed, H., Ahmad, N. B. H., and Shamsuddin, S. M. H. (2014). Bijective soft set classification of student's learning styles. In 2014 8th. Malaysian Software Engineering Conference (MySEC), pages 289-294.

Paireekreng, W. and Prexawanprasut, T. (2015). An integrated model for learning style classification in university students using data mining techniques. In 2015 12th International Conference on Electrical Engineering/Electronics, Computer, Telecommunications and Information Technology (ECTI-CON), pages 1-5.

Popescu, E., Dascalu, M., Becheru, A., Crossley, S., and Trausan-Matu, S. (2016). Predicting student performance and differences in learning styles based on textual complexity indices applied on blog and microblog posts: A preliminary study. In 2016 IEEE 16th International Conference on Advanced Learning Technologies (ICALT), pages 184188.

Sales, G. L. (2010). Learning Vectors: Um Modelo de Avaliação da Aprendizagem em EaD Online Aplicando Métricas Não-Lineares. Tese de doutorado, Universidade Federal do Ceará, Fortaleza, CE, Brasil.

Sweta, S. and Lal, K. (2015). Web usages mining in automatic detection of learning style in personalized e-learning system. In Ravi, V., Panigrahi, B. K., Das, S., and Suganthan, P. N., editors, Proceedings of the Fifth International Conference on Fuzzy and Neuro Computing (FANCCO - 2015), pages 353-363, Cham. Springer International Publishing.

Zhong, S., Li, Y., Liu, Y., and Wang, Z. (2017). A computational investigation of learning behaviors in moocs. Computer Applications in Engineering Education, 25(5):693705 . 